\title{
CDNA-711002--2
}

FABRICATION OF TARGET FOIIS BY ROLLING TECHNIQUES*

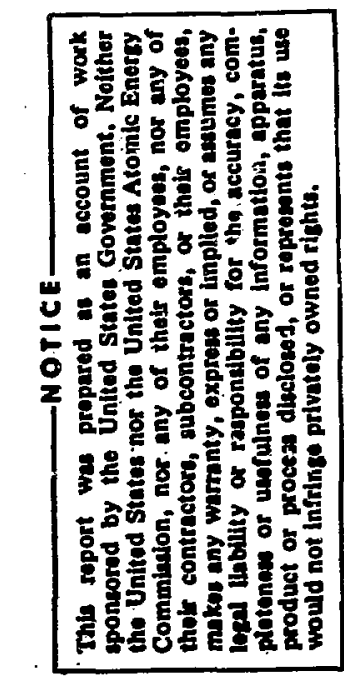

INTRODUCTION

Metallic foils of 1 to $10 \mu \mathrm{m}$ thickness are frequently used as targets in many nuclear reaction experiments. Commercial sources of high-quality foils in this thickness range are difficult to find, and experimenters are generally reguired to produce their own. Electroplating, vapor deposition, and rolling are some of the methods commonly used for the preparation of target foils. This paper is concerned only with the rolling technique of target preparation.

\section{FABRICATION EQUIPMENT}

Metal rolling has been defined basically as the deforming of a metal shape by means of a set of shaped rollers. This simple explanation has led to an erroneous belief that rolling can be accomplished easily by setting the roll gap to the thickness desired, passing the metal between the rolls, and retrieving the finished product on the other side. The truth of the matter is that metal rolling--especially the preparation of very thin metal foils--is a great deal more complicated than generally. assumed.

In the preparation of foils by rolling, consolidation and heat treatment equipment is required. Most natural metals can be obtained in a form suitable for rolling, but this is usually not true for isotopically enriched materials. Also, it is frequently necessary to consolidate scrap or jecovered metal powder when a variety of target sizes using a single material are being prepared from a limited amount of isotope. For consolidation, compaction dies and a hydraulic press are used. The pelletized materials require sintering and/or melting and casting prior to being rolled. Generally, a small laboratory-type arc furnace using a tungsten-tipped electrode and an inert gas atmosphere has been used at

*This work was performed under the auspices of the United States Atomic Energy Commission. 
Argonne National Laboratory (ANL) to melt and cast samples prior to rolling. Small quantities of metals (10 to $15 \mathrm{mg}$ ) have been successfully melted by this technique. The minimum quantity of metal that can be melted is directly dependent upon its density. Because precise temperature control is not possible, metals that have low melting points and/or high vapor pressures cannot be melted by this method. For these materials a small quartz, alumina, or metal tube is used that can be externally heated by either a resistance furnace or an induction coil. The containment tube should have the capability of being evacuated and backfilled with an inert gas. A furnace of this type is extremely versatile in that it can be used for sintering, melting, and heat-treating operations by varying the furnace geometry. A small muffle furnace is also required to heat specimens prior to hot rolling.

Laboratory or research rolling mills are used for foil fabrication. These machines have roll diameters ranging from 3 to 5 in. and roll face widths of 5 to 8 in. These mills have sufficient rigidity and power to enable one to roll a variety of materials. The addition of a smaller roll positioned between the larger rolls increases the capability of rolling materials to a thinner gauge and this method is currently being used to prepare target foils at ANL.

\section{PROCEDURE FOR THE PREPARATION OF ISOTOPIC MOLYBDENUM FOIIS}

Isotopically enriched molybdenum powder is pelletized and sintered in a hydrogen atmosphere at $1100^{\circ} \mathrm{C}$ for approximately $1 \mathrm{hr}$. After the partially sintered pellet is cool, it is arc melted on a water-sooled, copper hearth plate in a helium-argon atmosphere. The resultant metal bead is then encased in a stainless stael jacket of sufficient size to protect the molybdenum from cxidation during hot rolling. The jacket permits greater ease in handling and serves as an excellent heat sink.

The can (jacketed molybdenum bead) is preheated approximately 20 min in a muffle furnace at $1000 \mathrm{C}$ and is then passed through the rolling mill. Rolling reductions of 10 to $15 \%$ per pass are used during the initial rolling stages. After each third reduction pass, the sample is reheated for 5 to $10 \mathrm{~min}$. During these rolling stages, the can is alternately cross-rolled. After the thickness of the can has been reduced $50 \%_{\text {, }}$, the preheat temperature is decreased to $800^{\circ} \mathrm{C}$ and rolling reductions are reduced to 5-8\% per pass. The can is reheated after each reduction pass for about 3 min. Rolling is continued until the can is reduced to a thickness of about $0.5 \mathrm{~mm}(0.020 \mathrm{in}$.). After the final roll pass, the can is placed in the furrace and reheated for $20 \mathrm{~min}$ and then allowed to air cool. Most of the excess stainless steel is removed by cutting, and the remainder is removed by etching with hydrochloric acid. The resultant molybdenum foil is roughly circular in shape and approximately $0.2 \mathrm{~mm}$ thick. If the foil is $<0.2 \mathrm{~mm}$ in thickness, further rolling of the foil can be done at room temperature. Due to equipment limitations; it is not feasible to cold roll the molybdenum foil to a lower gauge size by direct rolling. Therefore, it is necessary to resort to the modified pack-rolling technique. The roling pack consists of a stainless steel sheet that is 
folded over to form an envelope with three open sides. This envelope or pack is nominally about $5 \mathrm{~cm}$ wide and 7 to $10 \mathrm{~cm}$ long. The molybdenum disk is inserted in. the stainless steel pack and cold rolled. Reductions of 5-10\% are taken per pass. During the course of the cold-rolling operation, new packs are used when necessary, and the foil is rotated so that its circular shape is retained. After decreasing the foil thickness approximately $70 \%$, the foil is removed from the pack and vacuum heat treated at $1000^{\circ} \mathrm{C}$ for $10 \mathrm{~min}$. The f'oil is trimmed to a rough square, and the coldrolling operations are repeated. This procedure is continued until the foil has reached a thickness of $2.5 \mathrm{mg} / \mathrm{cm}^{2}(2.5 \mu \mathrm{m})$, and the foil is then given a final heat treatment. A section of fojl having a known area is cut from the foil and weighed. This section can then be further cold rolled to the thickness required. Finally, areas of specific size are cut, measured, and weighed. Thickness adjustments are made by additional cold rolling. Foil thicknesses in the range of 200 to $250 \mathrm{\mu g} / \mathrm{cm}^{2}$ have been prepared using these procedures.

\section{GENERAL FOII-ROLIING CONSTDERATIONS}

It is most difficult, if not impossible, to outline an exact procedure that can be used for the preparation of all metal foils. The procedure will vary for different metals in accordance with mechanical properties, purity, and starting shape. Rolling equipment is also a factor in the selection of a suitable rulling procedure. One ean only state in gezeral terms that the sample is directly rolled as thin as practically feasible. The resultant foil is then further reduced by pack rolling. other steps, such as percent reduction per pass, heat treatments, and pack changes, neressary to the successful preparation of a target, must be determined by the fabricktor. The minimum rolled thickness for various metals will differ with their mechanical properties, density, and chemical reactivity.

Cleanliness in all procedures cannot be overstressed if suitable foils are to be produced. Dust particles on the foil surface, scratches, and fingerprints on the inner surfaces of the rolling packs will result in pinholes and/or tearing of the foil. Extreme care must be used during handling of the foil because ignition of the sample can occur readily in air.

\section{CONCLUSIONS}

The rolling method has been used to prepare a vast number of self-supporting foils during the past few years. It cannot completely replace other methods of foil preparation, but can make a greater number of foils available to the user. The major disadvantages of the prosess are as follows:

1. A considerable array of equipment is required.

2. Not all materials can be rolled to the thickness required. 
3. A considerable amount of time, effort, and patience must be expended to prepare a target of the required size.

These disadvantages are offset by the ability to:

1. Produce foils from minute quantities of starting material.

2. Reclaim a major portion of the scrap generated during foil fabrication.

3. Rework prtvious?y fabricated and used targets into new target foils with little loss of valuable material. 